\title{
RANDOM WALK ON THE SPEISER GRAPH OF A RIEMANN SURFACE
}

\author{
BY PETER G. DOYLE
}

\begin{abstract}
We consider the problem of determining the conformal type -hyperbolic or parabolic - of a covering surface of the Riemann sphere with $n$ punctures. To such a surface there corresponds a graph called the Speiser graph of the covering, and it is natural to ask for a criterion for the type of the surface in terms of properties of the graph. We show how to define a random walk on the vertices of the graph, so that the random walk is transient if and only if the surface is hyperbolic.
\end{abstract}

1. The type problem. A simply-connected open Riemann surface is conformally equivalent either to the open unit disk or to the entire complex plane [1]. In the first case the surface is said to be hyperbolic, or to have hyperbolic type; in the second case it is said to be parabolic. This dichotomy is extended to multiply-connected surfaces by declaring a surface to be hyperbolic if, like the unit disk, it has finite electrical resistance out to infinity, and parabolic if, like the plane, it has infinite resistance. Equivalently, a hyperbolic surface is one on which Brownian motion is transient, and a parabolic surface is one on which Brownian motion is recurrent $[\mathbf{8}, \mathbf{9}]$. The classical type problem for Riemann surfaces is the problem of determining whether a given open Riemann surface is hyperbolic or parabolic.

2. The Speiser graph of a covering surface. One special case of the type problem that has received a lot of attention is the problem of determining the type of an infinitely-sheeted covering surface of the Riemann sphere with $n$ punctures. Such a covering surface can be represented by a Speiser graph, as I will now describe.

Start by drawing a simple closed curve $C$ through the $n$ branch points, as shown in Figure 1. The branch points divide $C$ into $n$ segments, which we label $C_{1}, \ldots, C_{n}$. The curve $C$ divides the sphere into two parts, which we label $S_{a}$ and $S_{b}$. Cutting along the curves that cover $C_{1}, \ldots, C_{n}$ separates the covering surface into an infinite number of pieces, some that cover $S_{a}$ and some that cover $S_{b}$.

To reconstruct the surface, we must glue each copy of $S_{a}$ along each of the $n$ curves that form its boundary to one or another of the copies of $S_{b}$. The Speiser graph gives a recipe for carrying out these gluings. It is an infinite graph with vertices labelled $a$ and $b$ and edges labelled by integers between 1 and $n$. Each edge joins a vertex labelled $a$ to one labelled $b$. Each vertex has $n$ edges coming into it, labelled $1, \ldots, n$. The vertices labelled $a$ correspond to copies of $S_{a}$, and those labelled $b$ to copies of $S_{b}$. An edge labelled $i$ indicates

Received by the editors June 12, 1984 and, in revised form, June 29, 1984.

1980 Mathematics Subject Classification. Primary 30F20, 31A15, 60J15.

(C) 1984 American Mathematical Society $0273-0979 / 84 \$ 1.00+\$ .25$ per page 


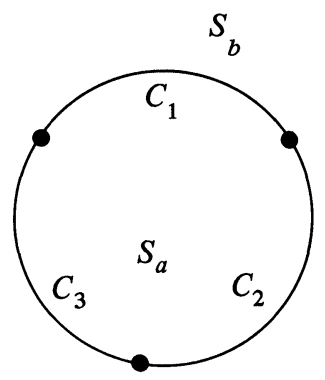

FIGURE 1

that the copies of $S_{a}$ and $S_{b}$ corresponding to the ends of the edge are to be glued along the parts of their boundaries that correspond to the curve $C_{i}$.

Examples of Speiser graphs are shown in Figures 2 and 3. Figure 2 shows the graph of the universal covering surface of the sphere with three punctures (the Riemann surface of the inverse of the modular function). Figure 3 shows the graph of the "class surface" of the sphere with three punctures, which is to homology what the universal covering is to homotopy; this surface is discussed by Lyons and McKean [11] and McKean and Sullivan [12]. For more examples, see Wittich [20].

Here is another way to look at the Speiser graph: Take the dual graph of the graph $C$, label it as shown in Figure 4, and take the inverse image under the covering projection. What you get is a copy of the Speiser graph sitting inside the covering surface. Note that a path of length two with edges labelled $i$ and $i+1$ (modulo $n$ ) corresponds to winding once around one of the branch points.

3. Determining the type of the surface from the Speiser graph. In introducing the Speiser graph, Speiser's idea $[18,19]$ was to determine the type of the surface from properties of the Speiser graph. Since the graph determines the covering surface up to the location of the punctures, and since moving the punctures around does not affect the type, the graph does in fact determine the type: the problem is to find a simple, natural, useful, sharp criterion expressed in terms of the graph itself. Many criteria were found that were simple, natural and useful $[\mathbf{1 7}, \mathbf{2 0}]$, but never one that was sharp. I am going to describe a criterion that is simple, natural and sharp, indicate how to prove it, and discuss how useful it is.

4. Random walk on the Speiser graph. Following McKean and Sullivan [12], we define a certain random walk on the vertices of the graph, which we call the McKean-Sullivan random walk. This is not the usual simple random walk, where the walker can move only to a neighboring vertex. Instead, we allow the walker to move from one vertex to any other vertex connected to it by a path in the graph that corresponds to winding some number of times - integral or half-integral - around one of the $n$ branch points. Such a 


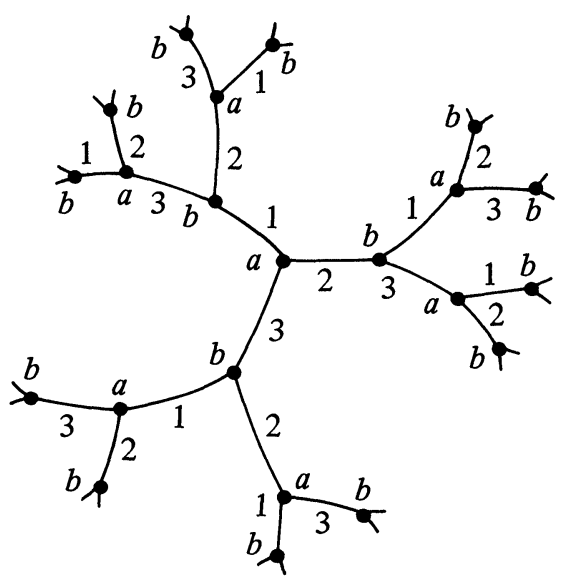

FigURE 2

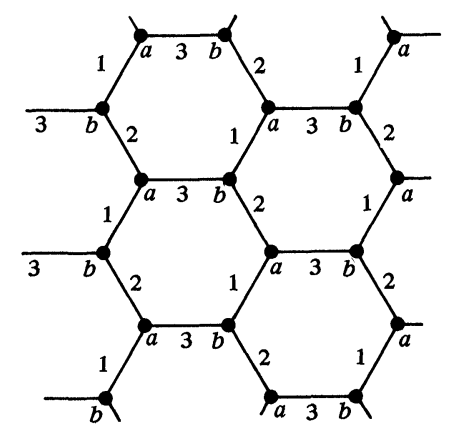

FIGURE 3

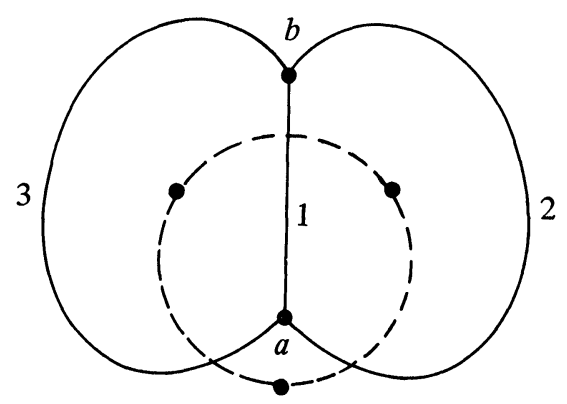

Figure 4 
path can be described in terms of the labelling as a path where the labels of the edges alternate between two integers that are consecutive modulo $n$. For our purposes, it does not matter what the exact values of the transition probabilities are, as long as they are symmetric and approximately Cauchydistributed: This means that the probability of a transition along a path of the kind we are considering must be the same forward or backward, and it must be on the order of $1 /\left(1+k^{2}\right)$, where $k$ is the length of the path.

THEOREM. The McKean-Sullivan random walk on the Speiser graph of a covering surface of the Riemann sphere with $n$ points removed is transient if and only if the surface is hyperbolic.

5. A probabilistic explanation and an electrical proof. This result is probabilistically obvious. The random walk on the graph is a discrete caricature of Brownian motion on the surface. The big jumps represent the fact that when the Brownian particle wanders in toward one of the punctures, it may wind quite a few times around the missing point before wandering back out to the graph again. We expect that the discrete caricature should be transient if and only if the Brownian motion is, that is, if and only if the surface is hyperbolic.

But while this result is probabilistically obvious, it is not at all clear how to prove it using purely probabilistic methods. The key step of the proof I am going to outline will depend on an analytic argument. The role of probability in this whole discussion may be likened to that of the stones in the fabulous stone soup.

The first step is to quadrangulate the punctured sphere, as indicated in Figure 5, and lift to get a quadrangulation of the covering surface. The graph consisting of the edges and vertices of this quadrangulation is an extension of the Speiser graph.

Now suppose we can shown that Brownian motion on the covering surface has the same type - transient or recurrent-as simple random walk on this extended Speiser graph, where the walker moves with probability $1 / n$ to each of its $n$ neighbors. To translate this information about random walk on the extended graph into information about random walk on the Speiser graph, we watch the random walk on the extended graph only when it is at points of the Speiser graph. If we do this, it will appear that the walker is carrying out a McKean-Sullivan random walk on the Speiser graph. But shutting our eyes when the walker is outside of the Speiser graph does not change the type of the random walk, so the McKean-Sullivan random walk is transient if and only if the surface is hyperbolic.

To complete the proof, we must justify the connnection between the type of Brownian motion on the surface and simple random walk on the extended Speiser graph. To do this, it is best to abandon probability and make an analytic argument, based on a method from the classical theory of electricity. This method, called Rayleigh's short-cut method, was introduced by Rayleigh [16] as a way of finding upper and lower bounds for the resistance of an electrical system; it is described at length in the monograph of Doyle and 


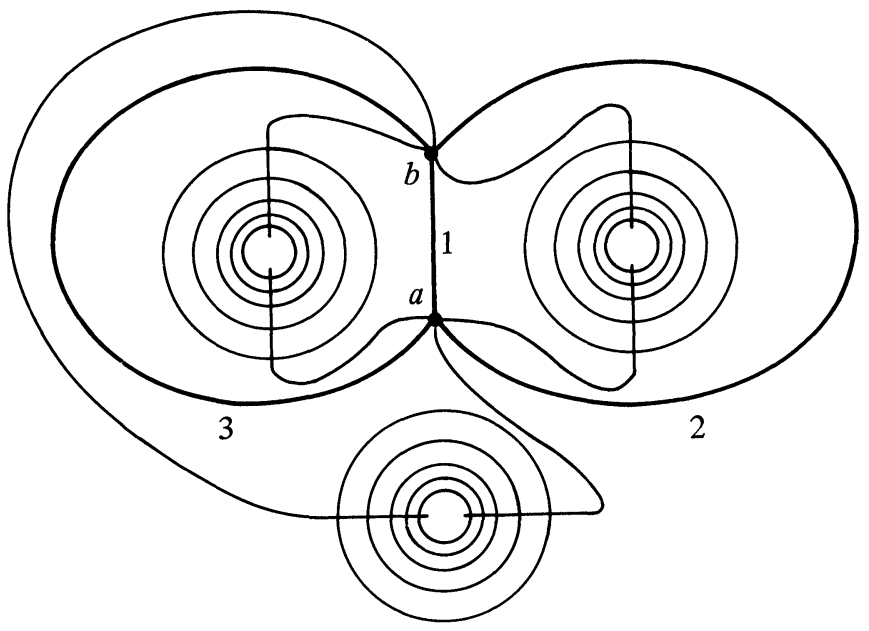

FigURE 5

Snell [4]. The idea of applying Rayleigh's method to the type problem is not new, going back at least to Royden's remarkable and much-overlooked thesis $[17]$.

The basis for the electrical argument is the observation that the Brownian motion is transient if and only if there is a system of currents out to infinity having finite dissipation rate. By a system of currents out to infinity, we mean a vector field for which, for a sufficiently large compact set, the vector field is divergenceless outside of the set, and the total flux through the boundary of the set is positive. The dissipation rate of the flow is the integral of the square of the current density, that is, the square of the Hilbert-space norm of the vector field.

Similarly, the random walk is transient if and only if there is a system of currents through the edges of the graph out to infinity having finite dissipation rate. Here, the dissipation rate is the sum of the squares of the currents through the edges.

To show that the graph and the surface have the same type - transient or recurrent-one shows how a system of currents could be transferred from the graph to the surface, or vice versa, without destroying the finiteness of the dissipation rate. The ingredients of the argument can be found in Royden's thesis $[\mathbf{1 7}]$.

Transferring a flow from the graph to the surface is easy. We simply fatten up the flow a little, taking the flow along each edge and spreading it over a narrow strip. Transferring a flow from the surface to the graph is a little trickier. The basic idea is to direct along each edge of the graph a current equal to the flux through the corresponding edge of the dual triangulation. The tricky part involves worrying about current that cuts across a corner of one of the polygons in the dual triangulation. For the original flow, the charge for doing this will be small, but for the transferred flow, the charge will be 
just as much as if the same amount of current had passed all the way across the polygon, and if we are not careful, we may find that the dissipation rate for the transferred flow has become infinite. Everything turns out all right, however, because for the extended Speiser graph, the polygons of the dual triangulation do not have too many sides, and are not too dissimilar; this was the reason for looking at the extended graph in the first place.

6. How useful is this criterion?. This result gives, I believe, a very satisfactory explanation of how the type of the surface is determined by properties of the Speiser graph. If this result is to be more than a curiosity, however, we must explain how we propose to tell whether the random walk we have defined is transient or recurrent. Otherwise, we merely have reduced one problem we cannot solve to another.

In certain cases we may be able to apply traditional probabilistic methods to determine whether the walk is transient or recurrent. For example, I suppose that one could give a thoroughly probabilistic proof that walk on the graph in Figure 3 is transient (though there is a better way to show this, as I will explain shortly). In general, though, traditional probabilistic methods do not seem to be much help.

So how do we tell if the walk is transient or recurrent? The answer is, translate the problem back into electrical terms and apply Rayleigh's method $[3,4,6,10,13]$. Taking this approach, it is easy to show that the graph in Figure 3 is transient, thus giving another proof of the theorem of Lyons, McKean, and Sullivan $[\mathbf{1 1}, \mathbf{1 2}]$ that the class surface of the sphere with three punctures is hyperbolic. This approach can also be used to derive known criteria for the type of the surface in terms of the rate of branching of the Speiser graph [20]; these now appear as conditions for recurrence or transience of the McKean-Sullivan random walk.

This suggests that what is really useful is not the result itself, but Rayleigh's method, the method by which the result is derived. The usefulness of Rayleigh's method is hardly news, however, because Rayleigh's method is equivalent $[\mathbf{5}, \mathbf{1 7}]$ to the method of extremal length, a geometrical method that has been the basis for most of the work on the type problem $[2,7,14,15,17$, 20].

This brings us to what may be the main use of this new criterion: It gives us yet another proof that the type problem is solved, and the method of extremal length is the solution. Not a formal proof, of course, but a proof in the sense of the dictum, "Prove all things, hold fast that which is good."

\section{REFERENCES}

1. W. Abikoff, The uniformization theorem, Amer. Math. Monthly 88 (1981), 574-592.

2. L. V. Ahlfors and L. Sario, Riemann surfaces, Princeton Univ. Press, Princeton, N.J., 1960.

3. P. G. Doyle, Application of Rayleigh's short-cut method to Polya's recurrence problem, Ph.D. Dissertation, Dartmouth College, Hanover, N.H., 1982.

4. P. G. Doyle and J. L. Snell, Random walks and electric networds, MAA Carus Monographs (to appear). 
5. R. J. Duffin, The extremal length of a network, J. Math. Anal. Appl. 5 (1962), 200-215.

6. D. Griffeath and T. M. Liggett, Critical phenomena for Spitzer's reversible nearest particle systems, Ann. Probab. 10 (1982), 881-895.

7. S. Kakutani, Applications of the theory of pseudo-regular functions to the type-problem of Riemann surfaces, Japanese J. Math. 13 (1937), 375-392.

8. - Two-dimensional Brownian motion and the type problem of Riemann surfaces, Proc. Japan. Acad. 21 (1949), 138-140.

9. __ Random walk and the type problem of Riemann surfaces, Contributions to the Theory of Riemann Surfaces, Princeton Univ. Press, Princeton, N.J., 1953, pp. 95-101.

10. T. J. Lyons, A simple criterion for transience of a reversible Markov chain, Ann. Probabl. 11 (1983), 393-402.

11. T. J. Lyons and H. P. McKean, Jr., Winding of the plane Brownian motion, Adv. in Math. 51 (1984), 212-225.

12. H. P. McKean Jr. and D. Sullivan, Brownian motion and harmonic functions on the class surface of the thrice-punctured sphere, Adv. in Math. 51 (1984), 203-211.

13. C. St. J. A. Nash-Williams, Random walk and electric currents in networks, Proc. Cambridge Philos. Soc. 55 (1959), 181-194.

14. R. Nevanlinna, Eindeutige analytische Funktionen, 2. Aufl., Springer, Berlin, 1953.

15. R. Osserman, Riemann surfaces of class A, Trans. Amer. Math. Soc. 82 (1956), 217245.

16. J. W. S. Rayleigh, On the theory of resonance, Collected Scientific Papers, Cambridge Univ. Press, Cambridge, 1899, pp. 33-75.

17. H. L. Royden, Harmonic functions on open Riemann surfaces, Trans. Amer. Math. Soc. 73 (1952), 40-94.

18. A. Speiser, Probleme aus dem Gebiet der ganzen transzendenten Funktionen, Comment. Math. Helv. 1 (1929), 289-312.

19. __ Ueber Riemannsche Flaechen, Comment. Math. Helv. 2(1930), 284-293.

20. H. Wittich, Neuere Untersuchungen ueber eindeutige analytische Funktionen, 2. Aufl., Springer, Berlin, 1968.

INSTITUTE FOR MATHEMATICS AND ITS APPLICATIONS, UNIVERSITY OF MiNNESOTA, MINNEAPOLIS, MINNESOTA 55455 